\title{
Fotosensibilización primaria por consumo de Ammi majus en bovinos: primer reporte en Argentina
}

\author{
Dorsch, M.A.; Cantón, G.J.; Odriozola, E.R. \\ Instituto Nacional de Tecnología Agropecuaria, EEA Balcarce, Ruta $226 \mathrm{~km} 73.5$, \\ Balcarce, Argentina. Tel: 02266 439100. E-mail: matiasdorsch@gmail.com
}

\begin{abstract}
Resumen
Dorsch, M.A.; Cantón, G.J.; Odriozola, E.R.: Fotosensibilización primaria por consumo de Ammi majus en bovinos: primer reporte en Argentina. Rev. vet. 29: 2, 137-138, 2018. Se describe un brote de fotosensibilización primaria en bovinos causado por el consumo de Ammi majus. Los animales manifestaron fotofobia, epífora, opacidad corneal bilateral y dermatitis en pezones, vulva y morro. Se descartó un origen secundario por daño hepático y se confirmó el diagnóstico por consumo voluntario de $A$. majus.
\end{abstract}

Palabras clave: bovino, fotosensibilización primaria, Ammi majus, falsa biznaga.

\begin{abstract}
Dorsch, M.A.; Cantón, G.J.; Odriozola, E.R.: Primary photosensitization in cattle due to consumption of Ammi majus: first report in Argentina. Rev. vet. 29: 2, 137-138, 2018. Primary photosensitization due to the consumption of Ammi majus was diagnosed in a bovine herd. Clinical signs included photophobia, epiphora, bilateral corneal opacity and dermatitis in udder, vulva and muzzle. A secondary photosensitization was ruled out and consumption of $A$. majus was diagnosed.
\end{abstract}

Key words: bovine, primary photosensitization, Ammi majus, Bishop's weed.

\section{INTRODUCCIÓN}

La dermatitis por fotosensibilización se desencadena cuando un compuesto fotodinámico se deposita en la piel expuesta a la luz solar. Teniendo en cuenta su origen se clasifica como (i) fotosensibilización primaria, cuando se ingieren sustancias fotodinámicas, (ii) fotosensibilización debido a un trastorno congénito (porfiria), por incapacidad de metabolizar adecuadamente los pigmentos del grupo heme (fotodinámicos) y (iii) fotosensibilización secundaria a una lesión hepática que impide la eliminación de la filoeritrina (pigmento fotodinámico) ${ }^{4}$.

Ammi majus es una especie vegetal de la familia de las Apiáceas, también llamada falsa biznaga, apio cimarrón, berro cimarrón y cicuta negra, capaz de causar fotosensibilización primaria en animales de producción. En Argentina suele ser consumida de forma preferencial por los bovinos, siendo más tóxica en esta especie que en otras ${ }^{8}$. Su toxicidad es mayor cuando la planta es consumida en estado reproductivo, ya que los principios fotodinámicos (furocumarinas) se concentran en las semillas ${ }^{2}$.

Hasta la fecha, en Argentina sólo existían reportes de intoxicaciones experimentales (Rev Med Vet 68: 98101; Vet Arg 7: 684-688), por lo cual el objetivo de este trabajo fue describir por primera vez un caso de intoxi-

Recibido: 4 abril 2018 / Aceptado: 27 julio 2018 cación natural de bovinos por $A$. majus en el partido de Coronel Suárez, Provincia de Buenos Aires, Argentina.

\section{MATERIAL Y MÉTODOS}

El episodio ocurrió en abril de 2015 en un establecimiento que contaba con 900 vacas de cruzas británicas que habían pastoreado durante una semana un rastrojo de trigo extensamente invadido por $A$. majus (Foto 1).

Se revisaron clínicamente los pacientes, tomándose muestras de sangre a 9 animales afectados, para realizar hemogramas completos y cuantificar la actividad

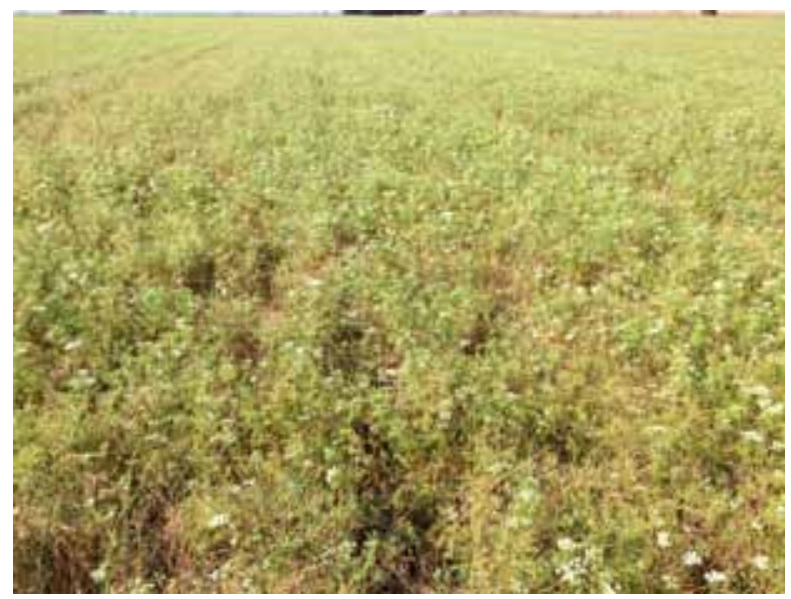

Foto 1: Rastrojo de trigo invadido por la falsa biznaga (A. majus). 


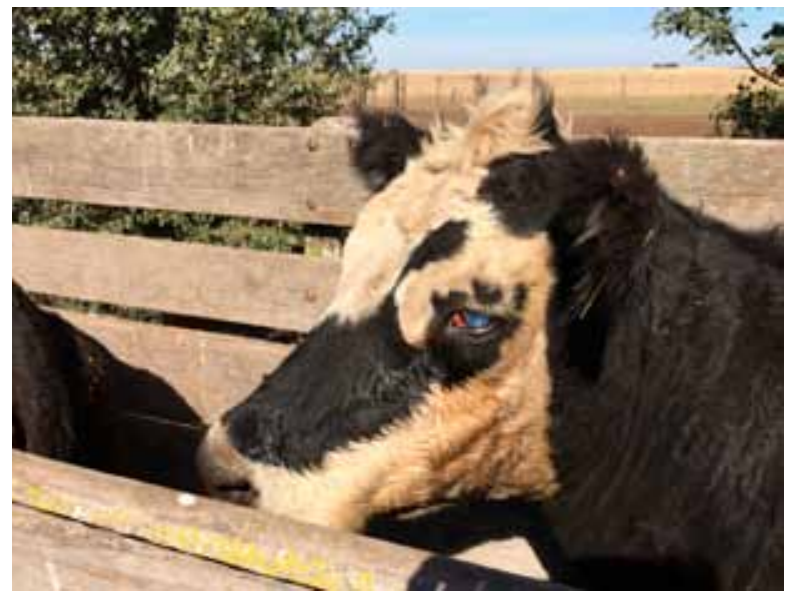

Foto 2: Opacidad corneal y epífora ocasionada por el consumo de $A$. majus.

de enzimas reveladoras de daño hepático: aspartato aminotransferasa (AST) y gamma-glutamil transferasa (GGT), utilizando un kit comercial (Wiener Lab, Rosario, Argentina). Además se recolectaron muestras de heces de animales afectados para detectar la presencia de semillas de $A$. majus.

\section{RESULTADOS Y DISCUSIÓN}

Aproximadamente el $40 \%$ de los animales mostró diferentes grados de fotofobia, epífora, opacidad corneal bilateral (Foto 2) y dermatitis en áreas despigmentadas y/o con escasa cobertura pilosa, signos similares a los descriptos en intoxicaciones experimentales ${ }^{1}$.

Aunque la morbilidad fue alta, cabe consignar que no murió ningún animal afectado, concordando con lo descripto por otros investigadores ${ }^{1}$. El consumo de $A$. majus se evidenció por la gran cantidad de semillas en las heces examinadas.

Los hemogramas y las actividades enzimáticas se encontraron dentro de los valores normales de referencia $^{3,5}$. Ello permitió descartar el origen hepatógeno de la afección, la cual cursa con consecuencias más gra- ves ${ }^{1,6,7}$. Asimismo, se descartó la presencia de porfiria congénita debido a la avanzada edad de los animales afectados y a la ausencia de alteraciones concordantes ${ }^{4}$.

Considerando su potencial tóxico y amplia difusión, A. majus debe incluirse en el diagnóstico diferencial ante cuadros de dermatitis por fotosensibilización como el descripto en este episodio.

\section{REFERENCIAS}

1. Dollahite JW, Younger RL, Hoffman GO. 1978. Photosensitization in cattle and sheep caused by feeding Ammi majus (greater Ammi; Bishop's-Weed). Am J Vet Res 39: 193-197.

2. Egyed MN, Shlosberg A, Eilat A, Malkinson M. 1975. Acute and chronic manifestations of Ammi majus induced photosensitisation in ducks. Vet Rec 97: 198-199.

3. Kramer JW. 2000. Normal hematology of cattle, sheep, and goats. In: Schalm's veterinary hematology (Feldman BF, Zinkl JG, Jain NC Ed), 5th ed., Lippincott Williams \& Wilkins, Philadelphia, p. 1075-1084.

4. Mauldin EA, Peters J. 2016. Integumentary system. In: Jubb, Kennedy and Palmer's Pathology of Domestic Animals (Maxie MG Ed), $6^{\text {th }}$ ed., Vol. 1, Saunders Elsevier, Philadelphia, USA, p. 577-579.

5. Meyer DJ, Harvey JW. 2000. Intervalos de referencia y tablas de conversión. En: El laboratorio en medicina veterinaria (Meyer DJ, Harvey JW Ed), 2nd ed., Inter-médica, Buenos Aires, p. 367-383.

6. Panter KE, Gardner DR, Lee ST, Pfister JA, Ralphs MH, Stegelmeier BL, James LF. 2007. Important poisonous plants in the United States. In: Veterinary toxicology (Gupta R Ed), 1st ed., Academic Press, London, p. 825872.

7. Rowe LD. 1989. Photosensitization problems in livestock. Vet Clinics of North America 5: 301-323.

8. Shlosberg A, Egyed MN, Eilat A. 1974. The comparative photosensitization properties of Ammi majus and Ammi visnaga in goslings. Avian Dis 18: 544-550. 